\title{
Homotopy Perturbation Method to Obtain Positive Solutions of Nonlinear Boundary Value Problems of Fractional Order
}

\author{
Hossein Jafari, ${ }^{1}$ Khadijeh Bagherian, $^{2}$ and Seithuti P. Moshokoa ${ }^{1,3}$ \\ ${ }^{1}$ Department of Mathematical Sciences, University of South Africa, Pretoria 0003, South Africa \\ ${ }^{2}$ Department of Mathematics, University of Mazandaran, Babolsar 47416-95447, Iran \\ ${ }^{3}$ Department of Mathematics and Statistics, Faculty of Science, Tshwane University of Technology, \\ Arcadia Campus, Building 2-117, Nelson Mandela Drive, Pretoria 0001, South Africa
}

Correspondence should be addressed to Hossein Jafari; jafari@umz.ac.ir

Received 6 March 2014; Accepted 7 May 2014; Published 15 June 2014

Academic Editor: Dumitru Baleanu

Copyright (C) 2014 Hossein Jafari et al. This is an open access article distributed under the Creative Commons Attribution License, which permits unrestricted use, distribution, and reproduction in any medium, provided the original work is properly cited.

We use the homotopy perturbation method for solving the fractional nonlinear two-point boundary value problem. The obtained results by the homotopy perturbation method are then compared with the Adomian decomposition method. We solve the fractional Bratu-type problem as an illustrative example.

\section{Introduction}

In the last three decades, extensive work has been done using the Adomian decomposition method (ADM) and the homotopy perturbation method (HPM), which provide analytical approximation for nonlinear differential equation [1-5]. These methods have been implemented in several boundary value problems with all types of boundary conditions. In the literature, nonlinear boundary value problems (BVP) have been studied extensively [4-9]. In the present paper, we obtain positive solutions of nonlinear fractional order BVP using the HPM. We investigate the following type of fractional BVP:

$$
\begin{aligned}
& D^{\alpha} u(x)+\mu F(x, u(x)) \\
& \quad=0, \quad 0<x<1,1<\alpha \leq 2, \mu>0, u(0)=0, u(1)=c,
\end{aligned}
$$

where $D^{\alpha}$ is Caputo fractional derivative, $c$ is a constant, $\mu>$ 0 , and $F:[0,1] \times[0, \infty) \rightarrow[0, \infty)$ is a continuous function. Bai and Lü have discussed existence and multiplicity results of positive solution of (1) by means of fixed-point theorems [10]. Jafari and Daftardar-Gejji have applied the ADM to obtain positive solution of the above equation [11].

In this paper, we employ the HPM to obtain positive solutions of (1) and then we compare the obtained result with those obtained by the Adomian decomposition method.

\section{Basic Definition}

Definition 1. A real function $f(x), x>0$ is said to be in the space $C_{\alpha}, \alpha \in R$, if there exists a real number $p(>a)$, such that $f(x)=x^{p} f_{1}(x)$ where $f_{1}(x) \in C[0, \infty)$. Clearly $C_{\alpha} \subset C_{\beta}$ if $\beta \leq \alpha$.

Definition 2. A function $f(x), x>0$, is said to be in the space $C_{\alpha}^{m}, m \in N \cup\{0\}$, if $f^{(m)} \in C_{\alpha}$.

Definition 3. The left sided Riemann-Liouville fractional integral of order $\mu \geq 0$ [12-14] of a function $f \in C_{\alpha}, \alpha \geq-1$ is defined as

$$
\begin{gathered}
I^{\mu} f(x)=\frac{1}{\Gamma(\mu)} \int_{0}^{x} \frac{f(t)}{(x-t)^{1-\mu}} d t, \quad \mu>0, x>0, \\
I^{0} f(x)=f(x) .
\end{gathered}
$$

Definition 4. Let $f \in C_{-1}^{m}, m \in N \cup\{0\}$. Then the (left sided) Caputo fractional derivative of $f$ is defined as $[1,12,14]$

$$
D^{\mu} f(x)= \begin{cases}I^{m-\mu} f^{(m)}(x) & m-1<\mu \leq m, m \in N, \\ \frac{d^{m} f(x)}{d x^{m}} & \mu=m .\end{cases}
$$


Note that $[1,12-14]$

$$
\begin{gathered}
I^{\mu} I^{\nu} f=I^{\mu+\nu} f, \quad \mu, v \geq 0, \\
f \in C_{\alpha}, \quad \alpha \geq-1, \\
I^{\mu} x^{\gamma}=\frac{\Gamma(\gamma+1)}{\Gamma(\gamma+\mu+1)} x^{\gamma+\mu}, \quad \mu>0, \quad \gamma>-1, \quad x>0, \\
I^{\mu} D^{\mu} f(x)=f(x)-\sum_{k=0}^{m-1} f^{(k)}(0) \frac{x^{k}}{k !}, \quad m-1<\mu \leq m .
\end{gathered}
$$

\section{Homotopy Perturbation Method}

In this section we employ the HPM to obtain positive solution of nonlinear fractional order BVP (1). We construct the following homotopy for (1):

$$
\mathscr{H}(v ; p)=D^{\alpha} u(x)+p \mu F(x, u(x))=0 .
$$

The embedding parameter $p$ monotonically increases from 0 to 1 as the trivial problem $D^{\alpha} u(x)=0$ is continuously transformed to the original problem $D^{\alpha} u(x)+\mu F(x, u(x))=$ 0 . If $p=0$, then (5) becomes a linear equation

$$
D^{\alpha} u(x)=0
$$

and when $p=1$, then (5) turns out to be the original equation (1).

The HPM uses the embedding parameter $p$ as a "small parameter," and writes the solution of (5) as a power series of $p$; that is,

$$
v=v_{0}+v_{1} p+v_{2} p^{2}+\cdots,
$$

$$
\begin{aligned}
& F(x, v(x)) \\
& \quad=H_{0}\left(v_{0}\right)+H_{1}\left(v_{0}, v_{1}\right) p+H_{2}\left(v_{0}, v_{1}, v_{2}\right) p^{2}+\cdots,
\end{aligned}
$$

where $H_{j}\left(v_{0}, v_{1}, \ldots, v_{j}\right)$ depend upon $v_{0}, v_{1}, \ldots, v_{j}$. In view of $(8), H_{j}\left(v_{0}, v_{1}, \ldots, v_{j}\right)$ will be calculated like Adomian polynomials $[15,16]$

$$
H_{j}\left(v_{0}, v_{1}, \ldots, v_{j}\right)=\left.\frac{1}{j !} \frac{\partial^{j}}{\partial p^{j}} F\left(x, \sum_{i=0}^{j} v_{i} p^{i}\right)\right|_{p=0} .
$$

Substituting (7) and (8) into (5) leads to

$$
\begin{aligned}
\mathscr{H}(v ; p)= & \sum_{m=0}^{\infty} D^{\alpha} v_{m} p^{m} \\
& +p \mu \sum_{m=0}^{\infty} H_{m}\left(v_{0}, \ldots, v_{m}\right) p^{m}=0 .
\end{aligned}
$$

Now equating the terms with identical powers of $p$, we can obtain a series of equations of the following form:

$$
\begin{aligned}
& p^{0}: D^{\alpha} u_{0}=0, \quad u_{0}(0)=0, u_{0}^{\prime}(0)=\beta, \\
& p^{1}: D^{\alpha} u_{1}+\mu H_{0}\left(v_{0}\right)=0, \quad u_{1}(0)=0, u_{1}^{\prime}(0)=0, \\
& p^{2}: D^{\alpha} u_{2}+\mu H_{1}\left(v_{0}, v_{1}\right)=0, \quad u_{2}(0)=0, u_{2}^{\prime}(0)=0, \\
& p^{3}: D^{\alpha} u_{3}+\mu H_{2}\left(v_{0}, v_{1}, v_{2}\right)=0, \quad u_{3}(0)=0, u_{3}^{\prime}(0)=0 .
\end{aligned}
$$

It is obvious that the system of nonlinear equations in (11) is easy to solve and the components $v_{i}, i \geq 0$, of the homotopy perturbation method can be completely determined and the series solutions are thus entirely determined.

Setting $p=1$ results in the approximate solution of (7):

$$
u=\lim _{p \rightarrow 1} v=v_{0}+v_{1}+v_{2}+\cdots
$$

We can approximate the solution $u$ by accelerating $p=1$ and the truncated series:

$$
\varphi_{k}=\sum_{m=0}^{k-1} v_{m}, \quad \lim _{k \rightarrow \infty} \varphi_{k}=u(x),
$$

where $\beta=v^{\prime}(0)$ will be determined by applying suitable boundary conditions of (1).

\section{Illustrative Examples}

In this section, to give a clear overview of the HPM for fractional nonlinear BVP, we present the following examples. We apply the HPM and compare the results with the ADM.

Example 1. Consider the following nonlinear boundary value problem [11]:

$$
\begin{aligned}
& D^{\alpha} u+u^{2}(x)-x^{4}-2 \\
& \quad=0, \quad 1<\alpha \leq 2, \quad 0 \leq x \leq 1, \quad u(0)=0, \quad u(1)=1 .
\end{aligned}
$$

According to the modified HPM [17], we construct the following homotopy:

$$
D^{\alpha} v-2+p\left(v^{2}(x)-x^{4}\right)=0 .
$$

Substitution of (7) and (8) into (15) and then equating the terms with same powers of $p$ yield the following series of equations:

$$
\begin{aligned}
& p^{0}: D^{\alpha} v_{0}-2=0, \quad v_{0}(0)=0, \quad v_{0}^{\prime}(0)=\beta, \\
& p^{1}: D^{\alpha} v_{1}(x)-x^{4}+H_{0}=0, \quad v_{1}(0)=0, \quad v_{1}^{\prime}(0)=0, \\
& p^{2}: D^{\alpha} v_{2}(x)+H_{1}=0, \quad v_{2}(0)=0, \quad v_{2}^{\prime}(0)=0,
\end{aligned}
$$

$$
p^{m+1}: D^{\alpha} v_{m+1}(x)+H_{m}=0, \quad v_{m}(0)=0, \quad v_{m}^{\prime}(0)=0,
$$

where $H_{m}$ are defined as

$$
H_{m}=\sum_{j=0}^{m} v_{j} v_{m-j}
$$

In view of (4) and (17), by applying the inverse operator $I^{\alpha}$ on both sides of (16) and solving corresponding integrals we get

$$
\begin{gathered}
v_{0}=x \beta+\frac{2 x^{\alpha}}{\alpha \Gamma(\alpha)}, \\
v_{1}=x \beta+\frac{2 x^{\alpha}}{\alpha \Gamma(\alpha)}-\frac{2 x^{\alpha+2} \beta^{2}}{\alpha\left(2+3 \alpha+\alpha^{2}\right) \Gamma(\alpha)}+\frac{24 x^{\alpha+4}}{\Gamma(\alpha+5)} \\
-\frac{4 x^{2 \alpha+1} \beta \Gamma(2+\alpha)}{\alpha \Gamma(\alpha) \Gamma(2+2 \alpha)}-\frac{8 x^{3 \alpha} \Gamma(2 \alpha)}{\Gamma(\alpha) \Gamma(1+\alpha) \Gamma(1+3 \alpha)},
\end{gathered}
$$




$$
\begin{aligned}
v_{2}= & x \beta+\frac{2 x^{\alpha}}{\alpha \Gamma(\alpha)}-\frac{2 x^{\alpha+2} \beta^{2}}{\alpha\left(2+3 \alpha+\alpha^{2}\right) \Gamma(\alpha)}+\frac{24 x^{\alpha+4}}{\Gamma(\alpha+5)} \\
& -\frac{4 x^{2 \alpha+1} \beta \Gamma(2+\alpha)}{\alpha \Gamma(\alpha) \Gamma(2+2 \alpha)}+\frac{8 x^{3+2 \alpha} \Gamma(4+\alpha)}{\left(\alpha+\alpha^{2}\right) \Gamma(\alpha) \Gamma(5+2 \alpha)} \\
& -\frac{48 x^{5+2 \alpha} \beta \Gamma(6+\alpha)}{\Gamma(5+\alpha) \Gamma(6+2 \alpha)}-\frac{8 x^{3 \alpha} \Gamma(2 \alpha)}{\Gamma(\alpha) \Gamma(1+\alpha) \Gamma(1+3 \alpha)} \\
& +\frac{16 x^{2+3 \alpha} \beta^{2} \Gamma(2+\alpha)}{\Gamma(\alpha) \Gamma(3+3 \alpha)}+\frac{16 x^{2+3 \alpha} \beta^{2} \Gamma(2+\alpha)}{\alpha \Gamma(\alpha) \Gamma(3+3 \alpha)} \\
& +\frac{8 x^{2+3 \alpha} \beta^{2} \Gamma(3+2 \alpha)}{\alpha \Gamma(\alpha) \Gamma(3+\alpha) \Gamma(3+3 \alpha)} \\
& -\frac{96 x^{4+3 \alpha} \Gamma(5+2 \alpha)}{\alpha \Gamma(\alpha) \Gamma(5+\alpha) \Gamma(5+3 \alpha)} \\
& +\frac{16 x^{1+4 \alpha} \beta \Gamma(2 \alpha)}{\Gamma(\alpha) \Gamma(1+\alpha) \Gamma(2+4 \alpha)} \\
& +\frac{48 x^{1+4 \alpha} \alpha \beta \Gamma(2 \alpha)}{\Gamma(\alpha) \Gamma(1+\alpha) \Gamma(2+4 \alpha)} \\
& +\frac{16 x^{1+4 \alpha} \beta \Gamma(2+3 \alpha)}{\Gamma(\alpha) \Gamma(2+2 \alpha) \Gamma(2+4 \alpha)} \\
& +\frac{16 x^{1+4 \alpha} \beta \Gamma(2+3 \alpha)}{\alpha \Gamma(\alpha) \Gamma(2+2 \alpha) \Gamma(2+4 \alpha)} \\
& \frac{32 x^{5 \alpha} \Gamma(2 \alpha) \Gamma(1+4 \alpha)}{\Gamma(\alpha) \Gamma(1+\alpha)^{2} \Gamma(1+3 \alpha) \Gamma(1+5 \alpha)} .
\end{aligned}
$$

Other components are determined similarly. Further we compute $u(x)$ for various values of $\alpha$.

For $\alpha=1.2$,

$$
\begin{aligned}
& u(x)=l_{1} \approx 1.81521 x^{1.2}-0.734086 x^{3.6}+0.141671 x^{5.2} \\
&+0.316924 x^{6}-0.0458495 x^{7.6}+x \beta \\
&-0.868184 x^{3.4} \beta+0.57265 x^{5.8} \beta-0.0260918 x^{7.4} \beta \\
&-0.257842 x^{3.2} \beta^{2}+0.345771 x^{5.6} \beta^{2} \\
&+0.0697577 x^{5.4} \beta^{3}
\end{aligned}
$$

For $\alpha=1.5$,

$$
\begin{aligned}
u(x)=l_{2} & \approx 1.50451 x^{1.5}-0.259467 x^{4.5}+0.0833665 x^{5.5} \\
& +0.0400539 x^{7.5}-0.0105982 x^{8.5}+x \beta \\
& -0.416667 x^{4} \beta+0.101256 x^{7} \beta-0.0077381 x^{8} \beta \\
& -0.171943 x^{3.5} \beta^{2}+0.0866187 x^{6.5} \beta^{2}+0.025 x^{6} \beta^{3} .
\end{aligned}
$$

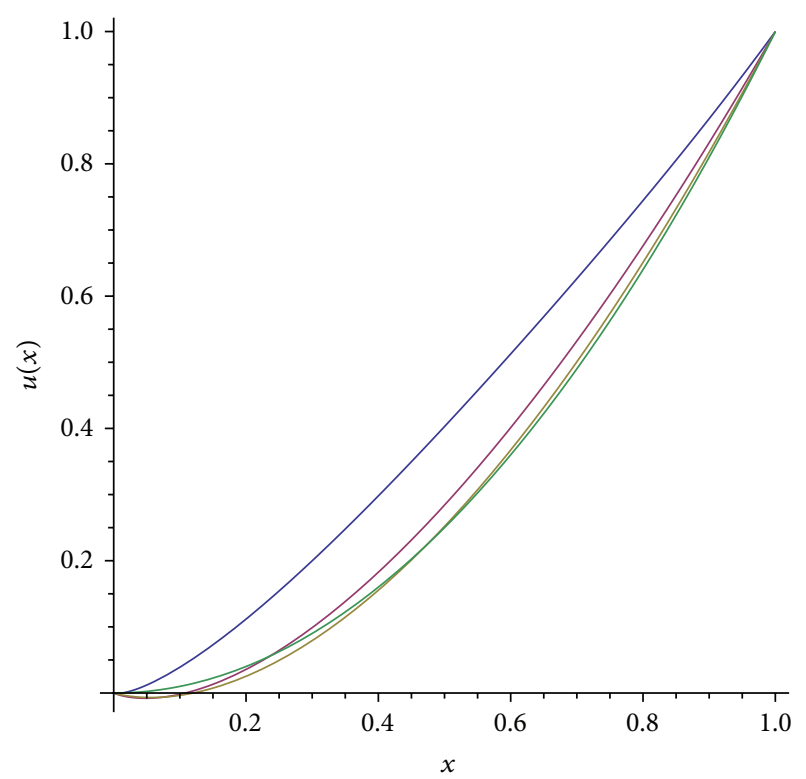

FIGURE 1

For $\alpha=1.7$,

$$
\begin{aligned}
& u(x)=l_{3} \approx 1.2947 x^{1.7}-0.119284 x^{5.1}+0.0580541 x^{5.7} \\
&+0.008744 x^{8.5}-0.0037705 x^{9.1}+x \beta \\
&-0.242159 x^{4.4} \beta+0.0285576 x^{7.8} \beta \\
&-0.00335666 x^{8.4} \beta-0.129606 x^{3.7} \beta^{2} \\
&+0.0320066 x^{7.1} \beta^{2}+0.0121972 x^{6.4} \beta^{3} .
\end{aligned}
$$

For $\alpha=2$,

$$
\begin{aligned}
u(x)= & l_{4} \approx x^{2}+x \beta-\frac{x^{5} \beta}{10}+\frac{x^{9} \beta}{360}-\frac{x^{4} \beta^{2}}{12} \\
& +\frac{11 x^{8} \beta^{2}}{1680}+\frac{x^{7} \beta^{3}}{252} .
\end{aligned}
$$

To determine $\beta$, we impose the boundary condition at $x=1$ and using $u(1)=1$, then

$$
\begin{array}{cc}
\beta=-0.757698 & \text { for } \alpha=1.2, \\
\beta=-0.493564 & \text { for } \alpha=1.5, \\
\beta=-0.29346 & \text { for } \alpha=1.7, \\
\beta=0 \quad \text { for } \alpha=2 .
\end{array}
$$

In Figure 1 we plot $l_{1}, \ldots, l_{4}$.

It should be remarked that the graphs drawn here using the HPM are in excellent agreement with those drawn using the ADM [11]. 


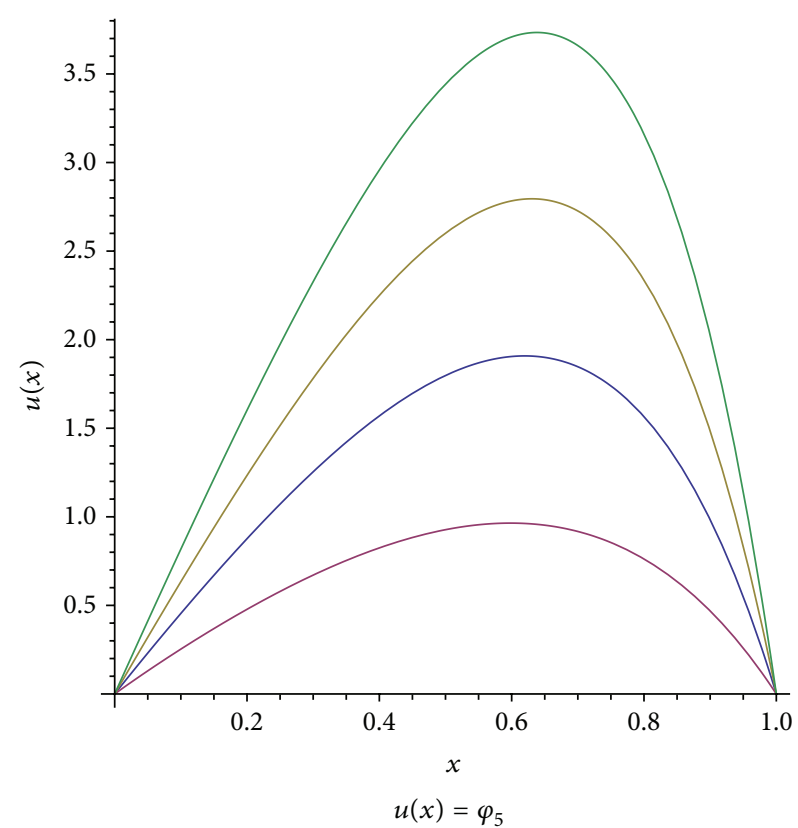

FIGURE 2

Example 2. Consider the one-dimensional fractional planar Bratu-type problem [4]

$$
\begin{gathered}
D^{\alpha} u(x)+\mu e^{u(x)}=0, \\
0 \leq x \leq 1, \quad 1<\alpha \leq 2, \quad u(0)=0, \quad u(1)=0 .
\end{gathered}
$$

In view of (5) we construct the following homotopy:

$$
\mathscr{H}(v ; p)=D^{\alpha} v(x)+p \mu e^{v(x)}=0 .
$$

Substituting (7) and (8) into (25) and proceeding as before we have

$$
\begin{gathered}
p^{0}: D^{\alpha} v_{0}=0, \quad v_{0}(0)=0, \quad v_{0}^{\prime}(0)=\beta, \\
p^{m+1}: D^{\alpha} v_{m+1}(x)+\mu H_{m}=0, \quad v_{m}(0)=0, \quad v_{m}^{\prime}(0)=0,
\end{gathered}
$$

where the first few Adomian polynomials $H_{n}$ that represent the nonlinear term $e^{v(x)}$ are defined as

$$
\begin{aligned}
& H_{0}=e^{v_{0}}, \\
& H_{1}=e^{v_{0}} v_{1}, \\
& H_{2}=\frac{e^{v_{0}} v_{1}^{2}}{2}+e^{v_{0}} v_{2} .
\end{aligned}
$$

In Figure 2 we plot $l_{i}, i=1,2,3,4$ where $l_{i}$ are as defined in Example 2 and $\mu=1$.

Remark 5. The graphs drawn in Figure 2 are in excellent agreement with those drawn in [11] using the ADM.
Theorem 6. The homotopy perturbation method for solving nonlinear fractional BVP (1) is Adomian's decomposition method with the homotopy $\mathscr{H}(v ; p)$ given by

$$
\mathscr{H}(v ; p)=D^{\alpha} v(x)+p \mu F(x, v(x)) .
$$

Proof. Substituting (7) and (8) into (5) and equating the terms with the identical powers of $p$, we have

$$
\begin{array}{r}
\mathscr{H}(v ; p)=\sum_{i=0}^{\infty} D^{\alpha} v_{i} p^{i}+p \mu \sum_{i=0}^{\infty} H_{i} p^{i}=0, \\
p^{0}: D^{\alpha} v_{0}=0, \quad v_{0}(0)=0, v_{0}^{\prime}(0)=\beta, \\
p^{n+1}: D^{\alpha} v_{n+1}+\mu H_{n}=0, \\
v_{n}(0)=0, \quad v_{n}^{\prime}(0)=0, \quad n=0,1,2, \ldots
\end{array}
$$

Applying the inverse operator $I^{\alpha}$ on both sides of (30), we have

$$
\begin{gathered}
v_{0}=\beta x \\
v_{n+1}=\mu I^{\alpha} H_{n}, \quad n=0,1,2, \ldots .
\end{gathered}
$$

We know $H_{n}=A_{n}$. Substituting (31) in (7) leads us to

$$
v=v_{0}+v_{1} p+v_{2} p^{2}+\cdots=\beta x+I^{\alpha} A_{0} p+I^{\alpha} A_{1} p^{2}+\cdots .
$$

So

$$
\begin{aligned}
\lim _{p \rightarrow 1} v & =\beta x+I^{\alpha} A_{1}+I^{\alpha} A_{2}+\cdots \\
& =\beta x+I^{\alpha} \sum_{i=0}^{\infty} A_{i}=\sum_{i=0}^{\infty} u_{i}=u .
\end{aligned}
$$

Therefore, by letting

$$
\mathscr{H}(v ; p)=v-f(x)-p N(v),
$$

we observe that the power series $v_{0}+v_{1} p+v_{2} p^{2}+\cdots$ corresponds to the solution of the equation $\mathscr{H}(v ; p)=$ $D^{\alpha} v+p \mu F(x, v(x))=0$ and becomes the approximate solution of (1) if $p \rightarrow 1$. This shows that the homotopy perturbation method is Adomian's decomposition method with the homotopy $\mathscr{H}(v ; p)$ given by $(34)$. The proof of Theorem 6 is completed.

\section{Conflict of Interests}

The authors declare that there is no conflict of interests regarding the publication of this paper.

\section{References}

[1] H. Jafari, An Introduction To Fractional Differential Equations, Mazandaran University Press, 2013, (Persian).

[2] H. Jafari, K. Sayevand, H. Tajadodi, and D. Baleanu, "Homotopy analysis method for solving Abel differential equation of fractional order," Central European Journal of Physics, vol. 11, no. 10, pp. 1523-1527, 2013. 
[3] S. Kumar and O. P. Singh, "Numerical inversion of the abel integral equation using homotopy perturbation method," Zeitschrift Fur Naturforschung, vol. 65, pp. 677-682, 2010.

[4] A.-M. Wazwaz, "Adomian decomposition method for a reliable treatment of the Bratu-type equations," Applied Mathematics and Computation, vol. 166, no. 3, pp. 652-663, 2005.

[5] A. M. Wazwaz, "A reliable algorithm for obtaining positive solutions for nonlinear boundary value problems," Computers \& Mathematics with Applications, vol. 41, no. 10-11, pp. 1237-1244, 2001.

[6] G. Adomian and R. Rach, "Analytic solution of nonlinear boundary value problems in several dimensions by decomposition," Journal of Mathematical Analysis and Applications, vol. 174, no. 1, pp. 118-137, 1993.

[7] G. Adomian, M. Elrod, and R. Rach, "A new approach to boundary value equations and application to a generalization of Airy's equation," Journal of Mathematical Analysis and Applications, vol. 140, no. 2, pp. 554-568, 1989.

[8] O. P. Agrawal, "Solution for a fractional diffusion-wave equation defined in a bounded domain," Nonlinear Dynamics, vol. 29, no. 1-4, pp. 145-155, 2002.

[9] R. Metzler and J. Klafter, "Boundary value problems for fractional diffusion equations," Physica A. Statistical Mechanics and Its Applications, vol. 278, no. 1-2, pp. 107-125, 2000.

[10] Z. Bai and H. Lü, "Positive solutions for boundary value problem of nonlinear fractional differential equation," Journal of Mathematical Analysis and Applications, vol. 311, no. 2, pp. 495-505, 2005.

[11] H. Jafari and V. Daftardar-Gejji, "Positive solutions of nonlinear fractional boundary value problems using Adomian decomposition method," Applied Mathematics and Computation, vol. 180, no. 2, pp. 700-706, 2006.

[12] D. Baleanu, K. Diethelm, E. Scalas, and J. J. Trujillo, Fractional Calculus Models and Numerical Methods, vol. 3 of Series on Complexity, Nonlinearity and Chaos, World Scientific, 2012.

[13] I. Podlubny, Fractional Differential Equations, vol. 198 of Mathematics in Science and Engineering, Academic Press, San Diego, Calif, USA, 1999.

[14] N. T. Shawagfeh, "Analytical approximate solutions for nonlinear fractional differential equations," Applied Mathematics and Computation, vol. 131, no. 2-3, pp. 517-529, 2002.

[15] H. Jafari and S. Momani, "Solving fractional diffusion and wave equations by modified homotopy perturbation method," Physics Letters A, vol. 370, no. 5-6, pp. 388-396, 2007.

[16] H. Jafari, S. Ghasempoor, and C. M. Khalique, "A comparison between Adomian's polynomials and He's polynomials for nonlinear functional equations," Mathematical Problems in Engineering, vol. 2013, Article ID 943232, 4 pages, 2013.

[17] Z. M. Odibat, "A new modification of the homotopy perturbation method for linear and nonlinear operators," Applied Mathematics and Computation, vol. 189, no. 1, pp. 746-753, 2007. 


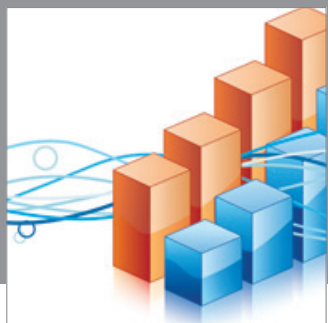

Advances in

Operations Research

mansans

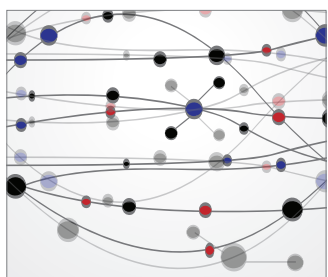

The Scientific World Journal
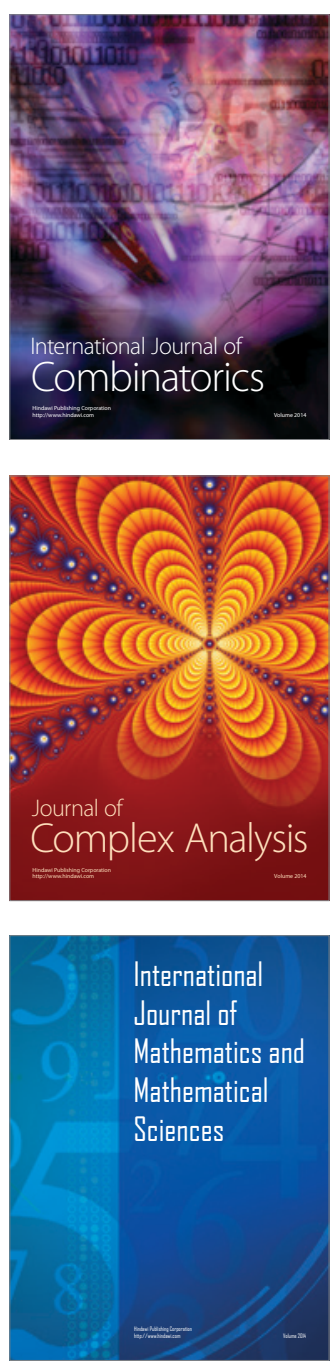
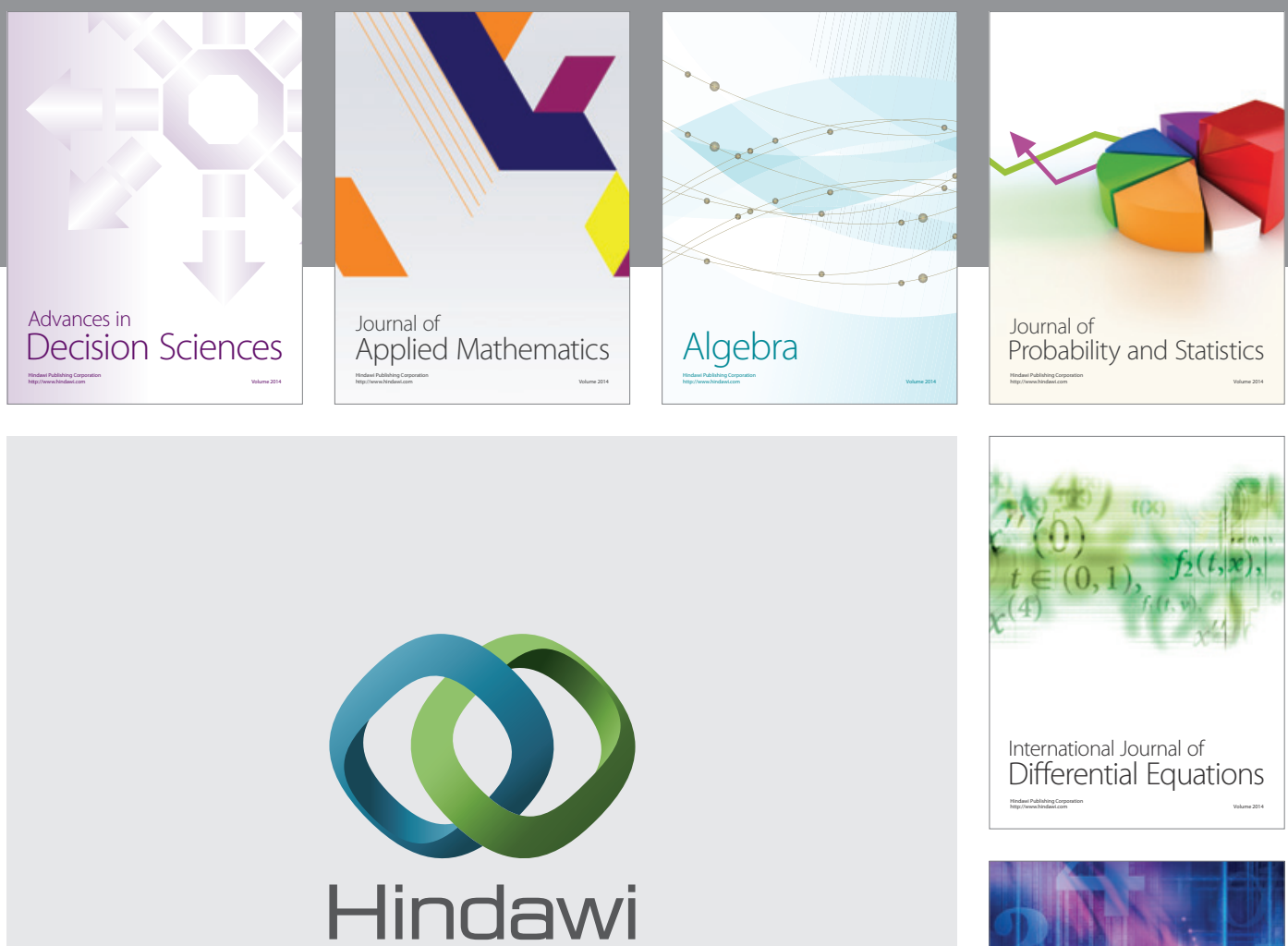

Submit your manuscripts at http://www.hindawi.com
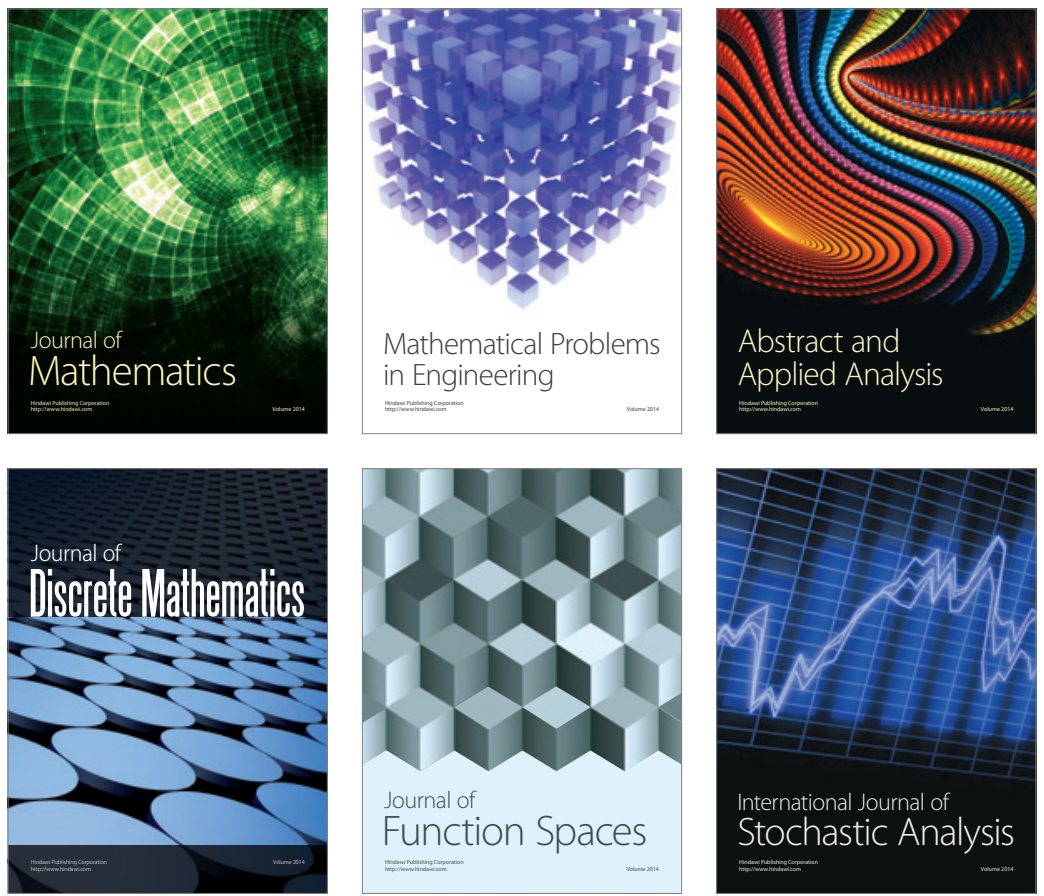

Journal of

Function Spaces

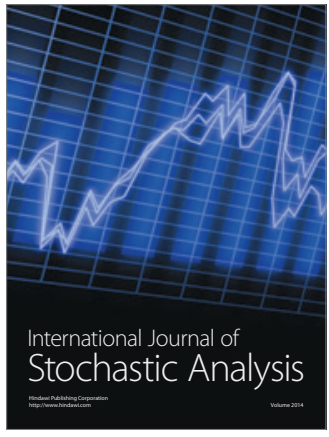

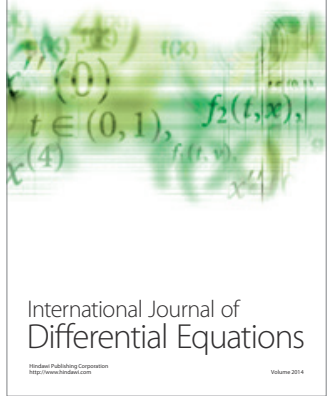
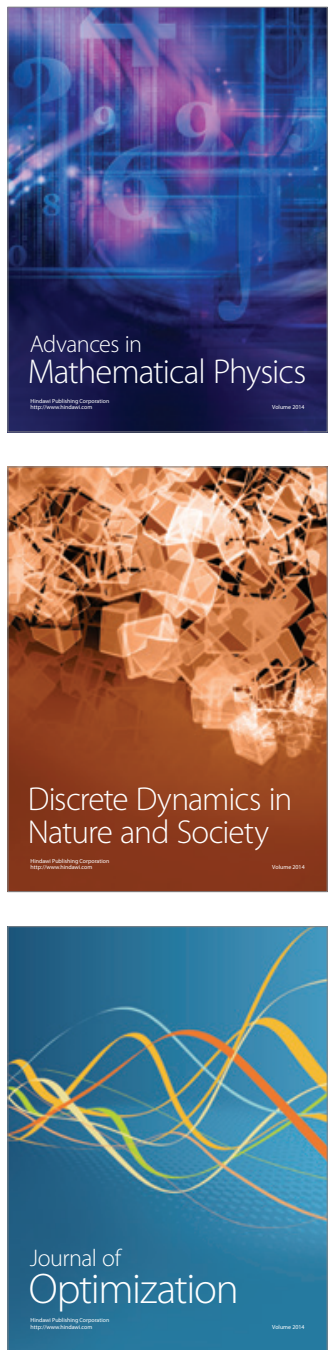